\title{
In-lab Replication of Saffran, Newport, \& Aslin (1996) Word segmentation: The role of distributional cues, Exp. 1
}

\author{
Lauren Skorb ${ }^{1} \&$ Joshua K. Hartshorne ${ }^{1}$ \\ ${ }^{1}$ Boston College
}

\begin{abstract}
These are the results for an in-lab replication of Saffran, Newport, \& Aslin (1996) Word segmentation: The role of distributional cues, Journal of Memory and Language, 35, 606621. This replication follows an online replication of the same experiment (Hartshorne 2017, Replication of Saffran, Newport, \& Aslin (1996) Word segmentation: The role of distributional cues, Exp. 1. PsyArXiv doi:10.17605/OSF.IO/E5C64).
\end{abstract}

Keywords: statistical learning, unsupervised learning, language acquisition, replication

\section{Introduction}

In this paper, we describe the methods and results for an in-lab replication of Experiment 1 from a classic statistical word-segmentation paper by Saffran, Newport, and Aslin (1996) (henceforth: SNA96). This experiment is part of a larger project to systematically replicate as many experiments involving statistical word learning in adults as possible and follows two online replications of the same study (Garcia et al., 2017; Hartshorne, 2017). The present study uses the materials from the second replication, and therefore all comparisons to our previous online replication refer to this second online replication (Hartshorne, 2017).

\section{Method}

\section{Subjects}

We ran a total of 100 subjects; 50 subjects in the partword condition (Ages 18-21, M=18.94) and 50 in the non-word condition (Ages 17-21, M=18.76). All but 7 subjects were native speakers of English and all were undergraduates recruited from a university psychology subject pool and compensated with course credit.

This total does not include excluded participants. In order to confirm that the results were not due to subjects who were not paying attention and to effectively follow the procedures from Hartshorne (2017) we excluded the 26 subjects (14 in the nonword condition and 12 in the partword condition)

Correspondence concerning this article should be addressed to Lauren Skorb, McGuinn Hall. E-mail: info@13atbc.org who missed any catch trials. Note that the catch trials were relatively subtle, so this is a strong filter.

\section{Materials \\ We used the same materials from our previ- ous online replication (Hartshorne, 2017).}

The language constructed for the experiment consisted of four consonants $(/ \mathrm{p} /, / \mathrm{t} /, / \mathrm{b} /, / \mathrm{d} /)$ and three vowels $(/ \mathrm{a} /, / \mathrm{i} /$, $/ \mathrm{u} /$ ) which, when combined, rendered an inventory of $12 \mathrm{CV}$ syllables. We combined eleven of these syllables (excluding /di/) to create six trisyllabic words: babupu, bupada, dutaba, patubi, pidabu, and tutibu. Some members of the syllable inventory occurred in more words than others; for example, /bu/ occurred in four words, while /ta/ occurred in only one. This ensured varying transitional probabilities within the words themselves.

We generated each syllable using MBROLA (Dutoit, Pagel, Pierret, Bataille, \& Van der Vrecken, 1996) with voice us1 and with full coarticulation between syllables. For each syllable, the consonant lasted for $100 \mathrm{~ms}$ and the vowel for $177 \mathrm{~ms}$, for a rate of 216 syllables/minute. We concatenated 252 tokens of each of the 6 words in a random order, with the stipulation that the same word never occurred twice in a row. We converted the resulting sound file from WAV to MP3 using SoX (Bagwell \& Contributors, 2015).

Average transitional probability within a word ranged from $42.0 \%$ to $100 \%$. Transitional probabilities between phonemes spanning words ranged from $5.0 \%$ to $59.0 \%$. 
For the test phase, we created 6 nonword foils and 6 partword foils. The nonwords (pibuda, badapu, tapubi, bubita, dubiti, bubibi) consisted of syllables from the language's syllable inventory which never followed each other in the speech stream, even across word boundaries. Thus, the transitional probabilities between each of the syllables in the nonwords were zero.

Each of the 6 partwords consisted of a syllable pair from a word from the language, plus an additional syllable. For example, the final syllable of the word pidabu was altered to create the partword pidata. Similarly, the first syllable of the word dutaba was altered to create the partword bitaba. Three partwords contained the first two syllables of words (pidata, bupabi, babuda) and three contained the final two syllables of words (bitaba, datubi, titibu).

We generated the stimuli for the test phase from the same syllables used to create the training, using the procedure described above. We created 72 test trials by combining each real word with each foil, with an approximately 500 ms gap in between, with the stipulation that the real word appeared first in half of the trials in each condition (nonword or partword).

In addition, we generated 6 catch trials, which pitted each word against a (different) foil that contained multiple phonemes that did not appear in the training.

\section{Apparatus}

We programmed the experiment using jsPsych (Leeuw, 2014) and ran participants individually in a closed room in our lab, allowing us to control the listening environment. Stimuli were presented with a Mac Book computer and Dell AX510 speakers.

\section{Procedure}

We tested subjects one at a time. Participants were instructed to listen to a "nonsense" language and were told that the language contained words, but no meanings or grammar. They were informed that their task was to figure out where the words began and ended. Subjects were given no information about the length or structure of the words or how many words the language contained. They were informed that the listening phase of the experiment consisted of three short blocks, followed by a test of their knowledge of the words in the language. Subjects were allowed to take a break after each of the first two 7-min listening blocks.

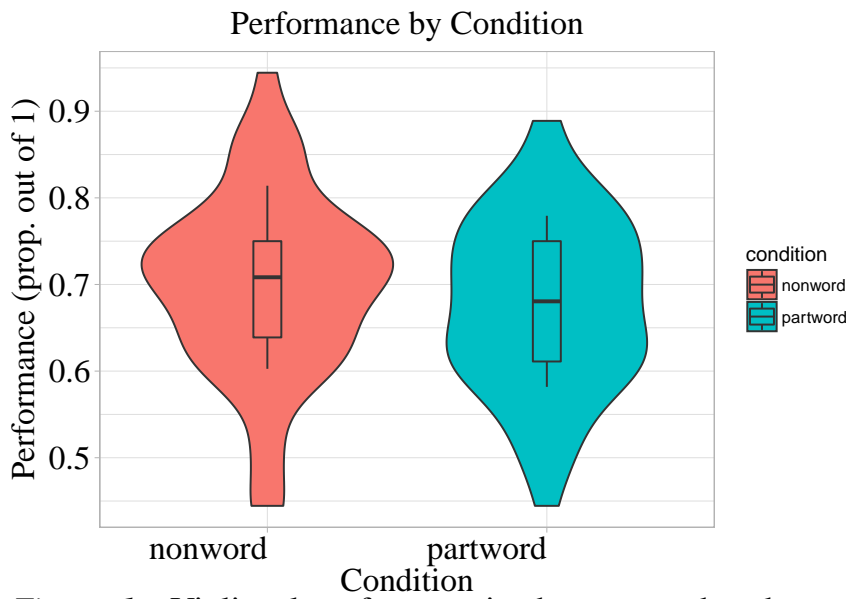

Figure 1. Violin plot of scores in the nonword and partword conditions in the present study. Note that only attentive subjects (who correctly answered all of the catch trials) are included. Note that the boxes represent the interquartile range, and the whiskers represent standard deviation.

After a total of $21 \mathrm{~min}$ of listening, subjects received a two-alternative forced-choice test. For each test item, subjects heard two trisyllabic strings, separated by $500 \mathrm{~ms}$ of silence. One of these strings was a word from the nonsense language, while the other was not. Subjects were asked to indicate which of the two strings sounded more like a word from the language by pressing either the " 1 " or " 2 " key on the keyboard. The test items were constructed by pairing the six words of the language with one of two types of trisyllabic foils. For half of the subjects, the six nonword foils were illegal strings of syllables from the language. For the other half of the subjects, the six foils were partwords, consisting of a syllable pair from a word plus an additional syllable. After the subject's response, the next item was presented. We randomized the order of the presentation of the items.

After the primary test trials, all subjects answered the 6 catch trials. The order was randomized for each subject. The catch trials followed the primary test trials immediately, without any overt distinction.

\section{Results}

\section{Original Analyses}

The overall results from the present replication are presented in Fig. 1. In the nonword test condition, the mean score was 25.18 of a possible $36(0.70, \mathrm{SE}=0.01)$, where chance performance equals 18. A single-sample t-test (two-tailed) showed overall performance significantly different from chance: $\mathrm{t}(49)=13.34, p=6.2 \mathrm{e}-18$. 6 of the 6 words (babupu, bupada, dutaba, patubi, pidabu, tutibu) in 
this condition were learned at a level significantly better than would be expected by chance $\left(p<0.05\right.$ for each word). ${ }^{1}$

Although the foils in the partword condition were designed to be more confusable with the words, the mean score for participants was 24.34 of a possible $36(0.68, \mathrm{SE}=0.01)$, a rate that significantly exceeded chance $(\mathrm{t}(49)=12.61, p=5.3 \mathrm{e}-17)$. Success did not significantly differ from that in the nonword condition $(\mathrm{t}(98)=1.14, p=0.26)$. 5 of the 6 words (babupu, dutaba, patubi, pidabu, tutibu) were learned significantly better than would be expected by chance $(p s<.01)$. Thus, we replicated the finding of statistical word segmentation, with similar rates to our previous replication $(\mathrm{M}=.65, \mathrm{SE}$ $=.02$ for nonword; $\mathrm{M}=.62, \mathrm{SE}=.02$ for partword).

Next, we asked whether the strengths of the transitional probabilities played a role in which words were learned better than others. Recall that the transitional probabilities between the pairs of syllables within words ranged between .43 and 1 . We split the six words into two sets, one set containing the three words with the highest average transitional probabilities (1.0, .77, and .75), and the other set containing the three words with the lowest average transitional probabilities $(.50, .43$, and .43). A matched-pairs t-test comparing subjects' mean performance on the three words with the highest transitional probabilities $(\mathrm{M}=0.70, \mathrm{SE}=0.01)$ to the three words with the lowest transitional probabilities $(\mathrm{M}=0.68, \mathrm{SE}=0.01)$ indicated no significant difference in performance on the words containing higher transitional probabilities: $\mathrm{t}(99)=0.76, p=0.45$. Thus, we do not replicate the original finding that words with higher transitional probabilities were more easily recognized, but we do replicate the non-significant finding from our online replication.

SNA96 report that accuracy was particularly high on partword trials with the foil bupabi. This is relevant because during training, the syllable pair /bupa/ was more common than any other. If subjects were simply following bigram frequencies, they should have relatively poor performance on this item. Similar to SNA96, we found that performance on such trials was higher than chance $(\mathrm{M}=0.71, \mathrm{SE}=0.03 ; \mathrm{t}(49)=7.61$, $p=7.7 \mathrm{e}-10)$. Thus, we replicated this original finding, but we do not replicate the findings from our online study, where we found that performance on such trials was low $(\mathrm{M}=.57, \mathrm{SE}=$ $.05)$ and indistinguishable from chance $(t(18)=1.29, p=.21)$.

These results may be probed further to determine which aspects of the words were learned. Recall that the partword test used two types of partwords. Half of the partwords contained the first two syllables of words plus a final syllable, and the other half contained the final two syllables of words plus an initial syllable. SNA96 found that while the first type produced false alarm rates significantly lower than chance $(\mathrm{M}=.29 ; t(11)=-61.3, p<.01)$, the latter did not $(\mathrm{M}=.48 ; t(11)<1)$, a difference that reached significance $(t(11)=3.25, p<.01)$. Our prior replication revealed below-chance false alarms for both change-final foils (M $=.36, \mathrm{SE}=.03, t(18)=4.90, p=.0001)$ and change-first foils $(\mathrm{M}=.41, \mathrm{SE}=.03, t(18)=2.88, p=.01)$, with no significant difference between the two $(t(18)=1.21, p=.24)$.

The present study replicates the results from our previous replication; we find below-chance false alarm rates for both change-final foils $(\mathrm{M}=0.32, \mathrm{SE}=0.02 \mathrm{t}(49)=-10.72, p=1.9 \mathrm{e}-$ 14) and change-first foils $(\mathrm{M}=0.33, \mathrm{SE}=0.02 \mathrm{t}(49)=-8.62$, $p=2.2 \mathrm{e}-11)$, with no significant difference between the two $(\mathrm{t}(49)=0.70, p=0.49)$. Thus, we were unable to replicate the original finding, but we successfully replicated our previous online replication.

\section{Revised Analyses}

There are two important limitations to the original analyses conducted by SNA96. First, their use of ttests on accuracy data violates core assumptions of t-tests: that the data are continuous and error is normally distributed. Violations of these assumptions can lead both to false positives and false negatives (Jaeger, 2008). Binomial models are more appropriate (Jaeger, 2008). ${ }^{2}$

Additionally, they treat items as fixed effects. Thus, any conclusions are specific to the specific stimuli used. No assessment of generalization can be made. Statisticians have suggested two options. The first is to conduct analyses separately over subject averages (as SNA96 did) and over item averages (Clark, 1973). The second option is to use mixed effects models (Baayen, Davidson, \& Bates, 2008).

Given these considerations, we re-analyzed the data using binomial mixed effects models, implemented in $\mathrm{R}$ using the lme4 package, ver. 1.1-12 (Bates et al., 2017). Our first model had a single fixed effect: foil type (nonword/partword). We considered two possible random effects structures. The first estimated separate random intercepts for subject and each word and foil separately, plus a random slope of foil type for each word. The second estimated random intercepts for subject and each word/foil pair. The first model failed to converge, so we use the second model and its random effects structure throughout.

${ }^{1}$ SNA96 do not state what statistical test was applied to the data for individual words. Because they used t-tests elsewhere, we assume they did so here as well.

${ }^{2} \mathrm{An}$ intermediate option is to transform accuracy measures to log-odds, though this method is less accurate than a binomial model. 
Thus, we assessed the effect of foil type with a binomial mixed effects model with a fixed effect of foil type and random effects as described above. The main effect of foil type was not significant (Wald's $z=-0.66, p=0.51$ ). ${ }^{3}$ These results match our results from the original analyses, therefore replicating both the original and replication results.

We further assessed evidence for learning by running the above model with no fixed effects. The intercept was significant when collapsing across conditions (Wald's $z=10.23, p=1.5 \mathrm{e}-24)$, or when considering the nonword and partword condition separately (respectively: Wald's $z=7.84, \quad p=4.4 \mathrm{e}-15$; Wald's $z=6.68, p=2.3 \mathrm{e}-11$ ), indicating that accuracy was significantly different from chance, which matches the results from the original analyses and replicates the original and replication results.

Next, we asked whether the three words with higher transition probability were recognized at a different rate from the three with lower transitional probability. For this model, we included a fixed effect of transitional probability and random effects as described above. The main effect of transitional probability (high vs. low) was not significant (Wald's $z=-0.53, p=0.59$ ). Similar to our results from the original analyses, we fail to replicate the original results, but successfully replicate our online replication results.

We also ran the model with no fixed effects, looking only at performance with the foil bupabi. We find that performance on these trials was significantly above chance (Wald's $z=4.37, p=1.2 \mathrm{e}-05$ ), and we therefore replicate the original effect of high accuracy on trials with the foil bupabi, which aligns with our results from the original analyses.

Finally, we compared false alarms to the partwords that matched real words on the first two syllables against accuracy for those that matched real words on the last two syllables with a model with a fixed effect for partword type and random effects as described above. The main effect of partword type was not significant (Wald's $z=-0.30, p=0.77$ ). Similar to our results from the original analyses, we fail to replicate the original results, but successfully replicate our online replication results.

\section{Comparison to Online Replication}

A central goal of this study was to determine whether the differences between the original study and the prior replications were due to venue (the original study was run in-lab, and our previous replications were run online through Amazon Mechanical Turk). In the present study, as with the prior replications, we replicated successful nonword

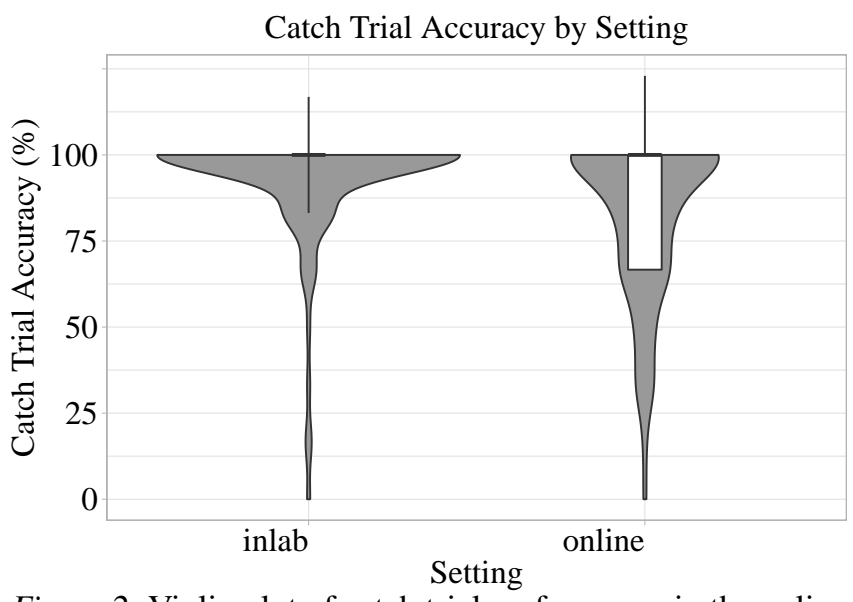

Figure 2. Violin plot of catch trial performance in the online and in-lab replications. Note that all subjects are included (exclusion criteria not applied). Note that the boxes represent the interquartile range, and the whiskers represent standard deviation.

and partword discrimination but did not replicate better discrimination in the nonword vs. the partword conditions, better discrimination of words with high transitional probabilities compared to low transitional probabilities, or better rejection of change-first vs. change-last foils. The one difference in terms of significance between the present in-lab replication and the previous online replications is that performance with the foil bupabi was significantly better than chance in the in-lab replications (and therefore replicating the finding from the original), but performance with the foil bupabi was not significantly different than chance in the online replications. Performance on these trials in the second online replication (we did not include this foil in the first online replication) was numerically greater than chance, but the difference did not reach significance. It is possible that this difference reached significance in the in-lab replications due to increased power (after exclusions, only 19 subjects heard the foil bupabi in the online replication). For convenience, we have summarized the results for all four experiments in Table 3 .

To further probe the effect of venue, we compare this replication to our previous online replication (Hartshorne, 2017) because the materials and methodology are identical with different venues. We were interested in first whether catch trial accuracy was better in the lab, indicating "better" subjects. Figure 2 shows the catch trial accuracy collapsed across conditions in both the present in-lab replication and previous online replication. We ran a

\footnotetext{
${ }^{3}$ Wald's $\mathrm{z}$ is somewhat anti-conservative when sample size is small [@luke2016evaluating]. Thus, these analyses may, if anything, overstate the evidence against the null.
} 
Table 1

Results of binomial mixed effects model of catch trial accuracy in online and in-lab replications. The model included the fixed effect of setting with random intercepts of subject and word/foil pair.

\begin{tabular}{lc}
\hline \hline & Overall Performance \\
\cline { 2 - 2 } & Performance \\
\hline Online & $-1.096^{* * *}(.184)$ \\
Constant & $2.721^{* * *}(.208)$ \\
\hline Observations & 1,356 \\
Log Likelihood & -451.284 \\
Akaike Inf. Crit. & 908.568 \\
Bayesian Inf. Crit. & 924.205 \\
\hline \hline Note: & ${ }^{*} \mathrm{p}<0.1 ;{ }^{* *} \mathrm{p}<0.05 ;{ }^{* * *} \mathrm{p}<0.01$ \\
& Parameter estimate $(\mathrm{SE})$
\end{tabular}

Test Phase Performance by Condition and Setting

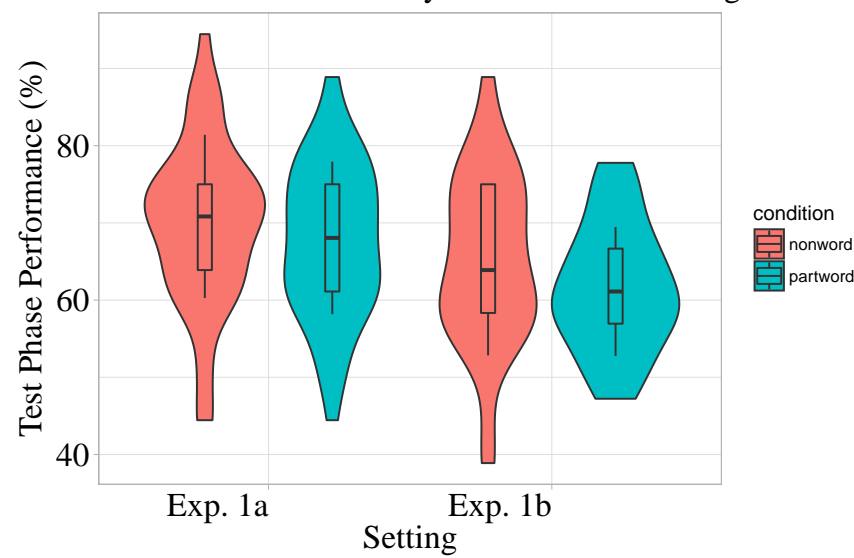

Figure 3. Violin plot of test phase performance broken up by condition in the online and in-lab replications. Note that only attentive subjects (who answered all catch trial questions correctly) are included. Note that the boxes represent the interquartile range, and the whiskers represent standard deviation.

mixed effects binomial regression with a fixed effect of setting (in-lab vs. online) and random intercept of word/foil pair ${ }^{4}$. We found that participants in the online replication performed significantly worse on the catch trials (Wald's $z=5.96, p=2.5 \mathrm{e}-09$; see Table 1), but Figure 2 shows that this difference is relatively small in terms of actual catch trial performance (Online mean catch trial performance: 0.83 ; in-lab mean catch trial performance: 0.94 ).

We also compared the statistical learning results using the subjects who passed the catch trials for both experiments. Figure 3 shows the total test phase accuracy across condition
Table 2

Results of binomial mixed effects model of test phase accuracy with fixed effects of condition and protocol and random intercepts of subject and word/foil pair. We centered the variables of condition and protocol, and due to the a priori hypothesis that performance would be better in the nonword condition and in-lab protocol, we made these two levels of the predictors positive 0.5 and the other levels (partword and online) -0.5. Note that only participants who passed all catch trials are included.

\begin{tabular}{lc}
\hline & Test Phase Accuracy \\
\cline { 2 - 2 } & Performance \\
\hline Online & $.264^{* * *}(.086)$ \\
condition & $.134(.153)$ \\
protocol:condition & $-.053(.171)$ \\
Constant & $.728^{* * *}(.076)$ \\
\hline Observations & 5,508 \\
Log Likelihood & $-3,376.188$ \\
Akaike Inf. Crit. & $6,764.377$ \\
Bayesian Inf. Crit. & $6,804.061$ \\
\hline \hline Note: & ${ }^{*} \mathrm{p}<0.1 ;{ }^{* *} \mathrm{p}<0.05 ;{ }^{* * *} \mathrm{p}<0.01$ \\
& Parameter estimate $(\mathrm{SE})$
\end{tabular}

and protocol (in-lab vs. online). We ran a binomial mixed effects model with fixed effects of condition and protocol and random intercepts of subject and word/foil pair. We centered the variables of condition and protocol, and due to the a priori hypothesis that performance would be better in the nonword condition and in-lab protocol, we made these two levels of the predictors positive 0.5 and the other levels (partword and online) -0.5. We found that the main effect of protocol type was significant (Wald's $z=3.08, p=2.1 \mathrm{e}-03)$, such that performance was significantly better in the in-lab replication than in the online replication. Condition (nonword vs. partword) was not a significant predictor. It is important to note that although the difference in performance between the in-lab and online replications is significant, Figure 3 shows that this difference is relatively small. More importantly, the theoretically important effects (see Table 3 for a review) remain largely unchanged from the online replications to the in-lab replications.

\footnotetext{
${ }^{4}$ The model failed to converge with random intercept of subject, so that random intercept was removed from the model
} 
Table 3

Effect Sizes Across Replications and Original

\begin{tabular}{cccccccc}
\hline Study & $\begin{array}{c}\text { Attn. } \\
\text { Screen }\end{array}$ & $\begin{array}{c}\text { Nonword } \\
\text { Discrimi- } \\
\text { nation }\end{array}$ & $\begin{array}{c}\text { Partword } \\
\text { Discrimi- } \\
\text { nation }\end{array}$ & $\begin{array}{c}\text { Non- v. } \\
\text { Partword }\end{array}$ & $\begin{array}{c}\text { High- vs. } \\
\text { LowTP }\end{array}$ & $\begin{array}{c}\text { Change- } \\
\text { first v. } \\
\text {-last }\end{array}$ & Bupabi \\
\hline Original & no & $26^{*}$ & $15^{*}$ & $11^{*}$ & $7 *$ & $19 *$ & $26^{*}$ \\
Online Rep. 1 & no & $7 *$ & $8^{*}$ & -1 & -1 & -8 & NA \\
Online Rep. 2 & no & $11^{*}$ & $7 *$ & 4 & -1 & 1 & 48 \\
Online Rep. 2 & yes & $15 *$ & $12^{*}$ & 3 & 0 & 5 & 43 \\
In-lab Rep. & no & $17^{*}$ & $15^{*}$ & 2 & 2 & 1 & $32^{*}$ \\
In-lab Rep. & yes & $20^{*}$ & $18^{*}$ & 2 & 2 & 2 & $29^{*}$ \\
\hline
\end{tabular}

Note: $\quad$ Effect sizes are reported as difference in percent correct in order to facilitate comparison with the original study. Regression coefficients can be found in the main text of this report and the report for prior replications. We do not report the results from the in-lab replication without an attention screen in this report but is included here for comparison to prior replications. Green represents a successfully replicated effect across all replications. $\quad *$ Significant at alpha level $\mathrm{p}=.05$ 


\section{References}

Baayen, R. H., Davidson, D. J., \& Bates, D. M. (2008). Mixedeffects modeling with crossed random effects for subjects and items. Journal of Memory and Language, 59(4), 390-412. Bagwell, C., \& Contributors, S. (2015). SoX: Sound eXchange, the swiss army knife of audio manipulation. sox.sourceforge.net.

Bates, D., Maeschler, M., Bolker, B., Walker, S., Christensen, R. H. B., Singmann, H., ... Green, P. (2017). Linear mixed-effects models using 'eigen' and s4. cran.rproject.org/web/packages/lme4/index.html.

Clark, H. H. (1973). The language-as-fixed-effect fallacy: A critique of language statistics in psychological research. Journal of Verbal Learning and Verbal Behavior, 12(4), 335359.

Dutoit, T., Pagel, V., Pierret, N., Bataille, F., \& Van der Vrecken, O. (1996). The mbrola project: Towards a set of high quality speech synthesizers free of use for non commercial purposes. In Proceedings of the Fourth International Conference on Spoken Language (Vol. 3, pp. 1393-1396).
IEEE.

Garcia, C. R., Iozzo, G. L., Lamirato, K. E., Ledoux, J. R., Mu, J., Murdock, K. N., ... Hartshorne, J. K. (2017). Replication of saffran, newport, \& aslin (1996) word segmentation: The role of distributional cues, exp. 1 . doi:10.17605/OSF.IO/QSYD2

Hartshorne, J. K. (2017). Replication of saffran, newport, \& aslin (1996) word segmentation: The role of distributional cues, exp. 1. doi:10.17605/OSF.IO/E5C64

Jaeger, T. F. (2008). Categorical data analysis: Away from ANOVAs (transformation or not) and towards logit mixed models. Journal of Memory and Language, 59(4), 434-446. doi:10.1016/j.jml.2007.11.007

Leeuw, J. R. de. (2014). JsPsych: A javascript library for creating behavioral experiments in a web browser. Behavior Research Methods, 47(1), 1-12.

Saffran, J. R., Newport, E. L., \& Aslin, R. N. (1996). Word segmentation: The role of distributional cues. Journal of Memory and Language, 35(4), 606-621. 